\title{
Solving the Puzzle of Cognitive Reserve Effects on Cognitive Decline: The Importance of Considering Functional Impairment
}

\author{
Andreas Ihle ${ }^{a, b, c}$ Élvio R. Gouveiab, d, e Bruna R. Gouveiab, e, f, g Dan Orsholits ${ }^{b, c}$ \\ Michel Oris ${ }^{b, c}$ Matthias Kliegel ${ }^{a, b}, c$ \\ ${ }^{a}$ Cognitive Aging Lab, Department of Psychology, University of Geneva, Geneva, Switzerland; ${ }^{b}$ Center for the \\ Interdisciplinary Study of Gerontology and Vulnerability, University of Geneva, Geneva, Switzerland; 'Swiss National \\ Centre of Competence in Research LIVES - Overcoming Vulnerability: Life course perspectives, Lausanne and Geneva, \\ Switzerland; dDepartment of Physical Education and Sport, University of Madeira, Funchal, Portugal; 'LARSyS, \\ Interactive Technologies Institute, Funchal, Portugal; ${ }^{f}$ Health Administration Institute, Secretary of Health of the \\ Autonomous Region of Madeira, Funchal, Portugal; '9Saint Joseph of Cluny Higher School of Nursing, Funchal, Portugal
}

\section{Keywords}

Cognitive decline $\cdot$ Cognitive reserve $\cdot$ Functional impairment $\cdot$ Old age $\cdot$ Longitudinal study

\begin{abstract}
Aims: We investigated whether the longitudinal relation between cognitive reserve accumulated across the lifespan and rate of cognitive decline over 6 years differed by the individual's degree of functional impairment. Methods: We analyzed longitudinal data from 897 older adults $(M=74.33$ years) tested on the Trail Making Test (TMT) in 2 waves 6 years apart. Results: There was a significant interaction of functional impairment with cognitive reserve on latent change in cognitive functioning. Specifically, with no functional impairment in the first wave of assessment, greater cognitive reserve accumulated across the lifespan significantly predicted a reduced cognitive decline over 6 years (i.e., smaller increase in TMT completion time). In contrast, with certain functional impairment (in at least some activities) in the first wave, greater cognitive reserve build-up predicted a steeper cognitive decline (i.e., larger increase in TMT completion time). Conclusion: Individuals with greater cognitive reserve accumulated across the lifespan show a re-
\end{abstract}

(C) 2020 S. Karger AG, Basel

www.karger.com/dem

Karger" duced cognitive decline if they still have relatively little functional impairment, while they will show a steeper decline (compared to individuals with less cognitive reserve) as soon as functional impairment becomes substantial.

(c) 2020 S. Karger AG, Base

\section{Introduction}

In the context of cognitive impairment such as dementia, cognitive reserve is a crucial factor [1-3]. In general, the cognitive reserve concept [4-6] aims to account for individual differences in the course of aging and neurodegenerative development. Cognitive reserve is built up during life through cognitive stimulation and serves later on as buffer for coping with brain alterations in order to preserve cognitive functioning in aging [4-6]. Frequently used proxies of accumulated cognitive reserve across the lifespan are education, cognitively demanding jobs, and leisure activity engagement [4-7]. Empirically, cross-sectional evidence demonstrated that these cognitive-reserve proxies are correlated with better cognitive functioning level in healthy older adults [7-9]. 
Yet, empirical findings on the longitudinal relation of cognitive reserve to long-term rate of cognitive decline are highly inconsistent and, therefore, remain inconclusive so far. For instance, some studies found relations of cognitive reserve markers (e.g., higher education and greater leisure activity engagement) to reduced cognitive decline [10-12], while others observed the opposite pattern, that is, steeper cognitive decline in individuals with greater cognitive reserve [13-16]. Yet, other studies found relations only to better cognitive performance level, but not to cognitive decline [17-20] (see [21-24] for discussions).

One avenue in solving this highly debated puzzle of inconsistent results concerns the question whether the relation between cognitive reserve and the rate of cognitive decline may be moderated by certain individual-difference characteristics. For example, in the context of severe cognitive decline and cognitive impairment, consequences with regard to functional impairment, such as difficulties in independently performing one's activities of daily living, are a crucial issue [25-27]. Interestingly, recent cross-sectional evidence suggests that correlations of cognitive-reserve markers and cognitive functioning level may be stronger in individuals with poorer, compared to those with better functional fitness status (such as strength, agility, and endurance) [28, 29]. However, so far research was only cross-sectional and considered rather momentary markers of cognitive reserve. To advance our understanding in this regard, a longitudinal investigation on long-term cognitive decline comprising the cumulative nature of cognitive reserve build-up during life is essential. Therefore, we investigated whether the longitudinal relation between cognitive reserve accumulated across the lifespan and rate of cognitive decline over 6 years as measured through performance changes in the Trail Making Test (TMT) differed by the individual's degree of functional impairment.

\section{Materials and Methods}

\section{Participants}

We analyzed data from 897 individuals who participated in the 2 waves of the Vivre-Leben-Vivere (VLV) survey [30-32]. Respondents were first interviewed during 2011 (Wave 1; W1) and again in 2017 (Wave 2; W2) using face-to-face computer-assisted personal interviewing (CAPI) and paper-pencil questionnaires. For further details regarding the rationale, design, recruitment, materials, and procedures of the VLV survey see [30-39]. Mean age of these respondents in $\mathrm{W} 1$ was 74.33 years $(\mathrm{SD}=6.50$, range $64-96)$; $51.4 \%$ were men.

\section{Materials}

In both waves, we administered the TMT parts A and B (TMT A and TMT B, respectively [40]). Functional impairment in W1 was indicated by difficulties in independently managing one's activities of daily living, such as dressing, feeding, washing, and transferring $[41,42]$. We used the Cognitive Reserve Index questionnaire [43] to assess proxies of accumulated cognitive reserve during life (comprising education, cognitive demand of jobs, and leisure activity engagement). To obtain an overall indicator of cognitive reserve accumulated across the lifespan, we computed a total cognitive reserve score (see $[43,44]$ for a detailed description). We controlled for the overall number of chronic diseases (such as heart diseases of ischemic or organic pathogenesis, primary arrhythmias, pulmonary heart diseases, hypertension, and peripheral vascular diseases) participants suffered from in W1 [45].

\section{Statistical Analyses}

Using latent change score modeling, we modeled latent cognitive factors of TMT completion time in W1 (constructed from TMT parts A and B in W1) and W2 (constructed from TMT parts $A$ and $B$ in W2) as well as a latent cognitive change variable regarding change in TMT completion time from W1 to W2 $[45,46]$. We included the following covariates to predict latent change: cognitive reserve, functional impairment in $\mathrm{W} 1$, the number of chronic diseases in $\mathrm{W} 1$, age in $\mathrm{W} 1$, sex, and the interaction of functional impairment in $\mathrm{W} 1$ with cognitive reserve (while taking the dependencies among all covariates into account). Data are available online as supplemental material (see online suppl. Table 1; see www. karger.com/doi/10.1159/000511768 for all online suppl. material). For model estimation, we used full information maximum likelihood.

\section{Results}

Greater functional impairment in W1 $(\beta=0.20, p<$ $0.001)$, a larger number of chronic diseases in W1 $(\beta=$ $0.10, p=0.012)$, and older age in $\mathrm{W} 1(\beta=0.30, p<0.001)$ significantly predicted a larger increase in TMT completion time from W1 to W2 (i.e., steeper cognitive decline). Sex and cognitive reserve total score per se did not predict changes in TMT completion time $(p>0.05)$. Yet, most importantly, there was a significant interaction of functional impairment with cognitive reserve total score $(\beta=$ $0.18, p<0.001)$.

We additionally explored this interaction pattern and disentangled the specific contributions by the 3 cognitive reserve markers. There was no interaction of functional impairment with education $(\beta=0.00, p=0.917)$. Notably, there were significant interactions of functional impairment with cognitive demand of jobs $(\beta=0.18, p<0.001)$ and of functional impairment with leisure activity engagement $(\beta=0.15, p<0.001)$.

To describe these interactions, we estimated in our latent change score model the longitudinal relation be- 
tween cognitive reserve and changes in TMT completion time at different degrees of functional impairment. Specifically, at an impairment score of zero in W1 (i.e., no functional impairment), greater cognitive reserve accumulated through leisure engagement significantly predicted a smaller increase in TMT completion time from W1 to W2 (i.e., reduced cognitive decline, $\beta=-0.15, p<$ $0.001)$. In contrast, at an impairment score of at least 2 points in W1 (i.e., incapability, i.e., entire dependence in at least 1 activity; or moderate impairment in at least 2 activities such as dressing, feeding, washing, or transferring [42]), greater cognitive reserve accumulated through leisure engagement significantly predicted a larger increase in TMT completion time from W1 to W2 (i.e., steeper cognitive decline, $\beta \geq 0.30, p<0.027$ ). Notably, already at an impairment score of at least 1 point in $\mathrm{W} 1$ (i.e., moderate impairment in at least 1 activity [42]), greater cognitive reserve accumulated through cognitive demand of jobs significantly predicted a larger increase in TMT completion time from W1 to W2 (i.e., steeper cognitive decline, $\beta \geq 0.26, p<0.001$ ).

\section{Discussion}

With regard to the highly inconsistent and debated puzzle of results observed in prior research on the relation between cognitive reserve and rate of cognitive decline, present longitudinal results have important implications. Using latent change score modeling (extracting measurement-error variance), we demonstrated that the individual's degree of functional impairment substantially moderates the longitudinal relation between cognitive-reserve built-up during life and cognitive decline over 6 years. Specifically, with no functional impairment in the first wave of assessment, greater cognitive reserve accumulated across the lifespan significantly predicted a reduced cognitive decline over 6 years (i.e., indicated by a smaller increase in TMT completion time). In contrast, with certain functional impairment (in at least some activities) in the first wave, greater cognitive-reserve build-up predicted a steeper cognitive decline (i.e., indicated by a larger increase in TMT completion time).

On first glance perhaps, the latter finding may sound counterintuitive. Yet, the overall pattern observed actually dovetails with the theoretical predictions of the cognitive reserve concept $[4,5]$ : individuals with greater cognitive reserve accumulated are able to tolerate more pathology, that is, they can still maintain cognitive functioning for a longer time and thereby show a reduced cognitive decline (though pathology is advancing). Cognitive functioning will begin to decline relatively late in time, after more pathology has accumulated. But, once decline begins, the cognitive system rapidly collapses and will then decline at a much steeper rate given that pathology has already been highly advanced $[4,5]$.

Importantly, our longitudinal observations empirically corroborate these conceptual predictions. Specifically, functional impairment seems to be a correlate or indicator of the mentioned ongoing pathology [25-27]. Present findings suggest that individuals with relatively little functional impairment may still be in a good overall condition in which also the cognitive system is still able to compensate the ongoing pathology and maintain cognitive functioning (i.e., thereby showing a reduced cognitive decline). In contrast, highly vulnerable individuals with considerable functional impairment may already suffer from substantial losses that are indicative of the upcoming terminal decline of the whole system [47, 48]. After reaching this critical developmental stage, these individuals will not be able to compensate anymore the upcoming breakdown of the cognitive system (not even by large cognitive reserve). As detailed above, especially individuals with greater cognitive reserve accumulated will then show a much steeper cognitive decline because they will drop from a much higher initial level (than those with less cognitive reserve who have started to decline already relatively early) $[4,5]$.

One may argue that our measure of functional impairment (that based on difficulties in independently managing one's activities of daily living), although being generic, yet could include a wide span of patients and some of them could be seriously compromised. To address this important issue, we controlled our analyses for chronic diseases participants suffered from and show that our findings are robust regarding such health-related confounders. Moreover, we demonstrate that already with a moderate functional impairment in some few activities (i.e., those individuals were still able to independently manage the majority of their other activities), the longitudinal relationship between greater cognitive reserve and steeper cognitive decline becomes significant. This suggests that this latter pattern may not be limited to individuals that are entirely dependent in all their activities of daily living. Future research might further scrutinize the role of additional factors including lifespan sociodemographic contexts, life events, personality, and age stereotypes that have been previously found to be important for cognitive aging [30, $33,49,50$ ] and that may play a role in further shaping the pattern observed in the present study. 
Our findings may appear somehow in contrast to recent cross-sectional studies in which cognitive functioning level among the more vulnerable individuals (i.e., those with poorer overall functional fitness status) seemed to more strongly depend on cognitive reserve $[28,29]$. Yet, these latter studies differ in several ways from the present one: their design was only cross-sectional and considered rather momentary markers of cognitive reserve. Moreover, those studies focused on a relatively young sample of older adults (about 70 years on average), which may still have been able to largely compensate their beginning losses (i.e., in these studies, findings were explained by cross-domain compensation effects, such as cognitive reserve still allowing to overcome the detrimental cognitive aftereffects of poor functional fitness status). In contrast, in the present study, our sample was on average already around 80 years at the second assessment - an age at which usually many individuals already show substantial losses [31], and for which functional impairment, such as difficulties in independently managing one's activities of daily living, may constitute a more pertinent issue $[41,42]$. The present longitudinal study helps to advance our knowledge in this regard and suggests that individuals with greater cognitive reserve accumulated across the lifespan show a reduced cognitive decline if they still have relatively little functional impairment, while they will show a steeper decline (compared to individuals with less cognitive reserve) as soon as functional impairment becomes substantial.

"Published in Celebration of the 30th Anniversary of the inception of Dementia and Geriatric Cognitive Disorders 1990-2020".

\section{Acknowledgements}

The authors are grateful to the Swiss National Science Foundation for its financial assistance. The authors also thank the participants of the VLV study, as well as all members of the LIVES project IP213 and LINK institute who contributed to the realization of the VLV study.

\section{Statement of Ethics}

All participants gave their written informed consent for inclusion in this study before participating. The present study was conducted in accordance with the Declaration of Helsinki, and this study protocol had been approved by the Ethics Commission of the Faculty of Psychology and Social Sciences of the University of Geneva (project identification codes: CE_FPSE_14.10.2010, and CE_FPSE_05.04.2017).

\section{Conflict of Interest Statement}

The authors have no conflicts of interest to disclose.

\section{Funding Sources}

This work was supported by the Swiss National Center of Competence in Research LIVES - overcoming vulnerability: life course perspectives, granted by the Swiss National Science Foundation (Grant No. 51NF40-185901). AI acknowledges support from the Swiss National Science Foundation (Grant No. 10001C_189407). E.R.G. and B.R.G. acknowledge support from LARSyS - Portuguese national funding agency for science, research, and technology (FCT) Pluriannual funding 2020-2023 (Reference: UIDB/50009/2020).

The funding sources had no role in the preparation of data or the manuscript. Moreover, the authors have not entered into an agreement with the funding organization that has limited their ability to complete the research as planned and publish the results. The authors have had full control of all the primary data.

\section{Author Contributions}

A.I., E.R.G., and B.R.G. formulated the research question, analyzed the data, and wrote the manuscript. D.O. assisted in writing. M.O. and M.K. formulated the research question, conceptualized the study, supervised the data collection, and participated in writing.

\section{References}

1 Karp A, Paillard-Borg S, Wang HX, Silverstein M, Winblad B, Fratiglioni L. Mental, physical and social components in leisure activities equally contribute to decrease dementia risk. Dement Geriatr Cogn Disord. 2006 Jan;21(2):65-73.

2 Paillard-Borg S, Fratiglioni L, Xu W, Winblad B, Wang HX. An active lifestyle postpones dementia onset by more than one year in very old adults. J Alzheimers Dis. 2012 Sep;31(4):835-42.
3 Perneczky R, Alexopoulos P, Schmid G, Sorg C, Förstl H, Diehl-Schmid J, et al. [Cognitive reserve and its relevance for the prevention and diagnosis of dementia]. Nervenarzt. 2011 Mar;82(3):325-35.

4 Stern Y. What is cognitive reserve? Theory and research application of the reserve concept. J Int Neuropsychol Soc. 2002 Mar;8(3): $448-60$.

Ihle/Gouveia/Gouveia/Orsholits/Oris/ Kliegel 
5 Stern Y. Cognitive reserve. Neuropsychologia. 2009 Aug;47(10):2015-28.

6 Stern Y. Cognitive reserve in ageing and Alzheimer's disease. Lancet Neurol. 2012 Nov; 11(11):1006-12.

7 Opdebeeck C, Martyr A, Clare L. Cognitive reserve and cognitive function in healthy older people: a meta-analysis. Neuropsychol Dev Cogn B Aging Neuropsychol Cogn. 2016 May;23(1):40-60

8 Ihle A, Gouveia ÉR, Gouveia BR, Freitas DL, Jurema J, Tinôco MA, et al. High-density lipoprotein cholesterol level relates to working memory, immediate and delayed cued recall in Brazilian older adults: the role of cognitive reserve. Dement Geriatr Cogn Disord. 2017 Jul;44(1-2):84-91.

9 Ihle A, Gouveia ÉR, Gouveia BR, Freitas DL, Jurema J, Machado FT, et al. The relation of hypertension to performance in immediate and delayed cued recall and working memory in old age: the role of cognitive reserve. J Aging Health. 2018 Sep;30(8):1171-87.

10 Newson RS, Kemps EB. General lifestyle activities as a predictor of current cognition and cognitive change in older adults: a cross-sectional and longitudinal examination. J Gerontol B Psychol Sci Soc Sci. 2005 May;60(3): P113-20.

11 Schneeweis N, Skirbekk V, Winter-Ebmer R. Does education improve cognitive performance four decades after school completion? Demography. 2014 Apr;51(2):619-43.

12 Wang HX, Jin Y, Hendrie HC, Liang C, Yang $\mathrm{L}$, Cheng Y, et al. Late life leisure activities and risk of cognitive decline. J Gerontol A Biol Sci Med Sci. 2013 Feb;68(2):205-13.

13 Hall CB, Derby C, LeValley A, Katz MJ, Verghese J, Lipton RB. Education delays accelerated decline on a memory test in persons who develop dementia. Neurology. 2007 Oct 69(17):1657-64.

14 Helzner EP, Scarmeas N, Cosentino S, Portet F, Stern Y. Leisure activity and cognitive decline in incident Alzheimer disease. Arch Neurol. 2007 Dec;64(12):1749-54.

15 Scarmeas N, Albert SM, Manly JJ, Stern Y. Education and rates of cognitive decline in incident Alzheimer's disease. J Neurol Neurosurg Psychiatry. 2006 Mar;77(3):308-16.

16 Thorvaldsson V, Skoog I, Johansson B. IQ as moderator of terminal decline in perceptual and motor speed, spatial, and verbal ability: testing the cognitive reserve hypothesis in a population-based sample followed from age 70 until death. Psychol Aging. 2017 Mar; 32(2):148-57.

17 Bielak AA, Anstey KJ, Christensen H, Windsor TD. Activity engagement is related to level, but not change in cognitive ability across adulthood. Psychol Aging. 2012 Mar;27(1) 219-28.

18 Tucker-Drob EM, Johnson KE, Jones RN The cognitive reserve hypothesis: a longitudinal examination of age-associated declines in reasoning and processing speed. Dev Psychol. 2009 Mar;45(2):431-46.
19 Van Dijk KR, Van Gerven PW, Van Boxtel MP, Van der Elst W, Jolles J. No protective effects of education during normal cognitive aging: results from the 6-year follow-up of the Maastricht aging study. Psychol Aging. 2008 Mar;23(1):119-30.

20 Vaughan L, Erickson KI, Espeland MA, Smith JC, Tindle HA, Rapp SR. Concurrent and longitudinal relationships between cognitive activity, cognitive performance, and brain volume in older adult women. J Gerontol B Psychol Sci Soc Sci. 2014 Nov;69(6):826-36.

21 Ritchie SJ, Tucker-Drob EM, Starr JM, Deary IJ. Do cognitive and physical functions age in concert from age 70 to 76 ? Evidence from the lothian birth cohort 1936. Span J Psychol. 2016 Dec;19:E90.

22 Ritchie SJ, Tucker-Drob EM, Cox SR, Corley J, Dykiert D, Redmond P, et al. Predictors of ageing-related decline across multiple cognitive functions. Intelligence. 2016 Nov-Dec; 59:115-26.

23 Bielak AA, Cherbuin N, Bunce D, Anstey KJ. Preserved differentiation between physical activity and cognitive performance across young, middle, and older adulthood over 8 years. J Gerontol B Psychol Sci Soc Sci. 2014 Jul;69(4):523-32.

24 Salthouse TA. Mental exercise and mental aging: evaluating the validity of the "use it or lose it" hypothesis. Perspect Psychol Sci. 2006 Mar;1(1):68-87.

25 Shin JH, Lim JY, Kim KW, Kim S, Lee J, Paik NJ. Functional and physical abilities in the early continuum of cognitive decline. Dement Geriatr Cogn Disord. 2015 Jan;39(1-2):41-51.

26 Rajan KB, Hebert LE, Scherr PA, Mendes de Leon CF, Evans DA. Disability in basic and instrumental activities of daily living is associated with faster rate of decline in cognitive function of older adults. J Gerontol A Biol Sci Med Sci. 2013 May;68(5):624-30.

27 Yeh YC, Lin KN, Chen WT, Lin CY, Chen TB, Wang PN. Functional disability profiles in amnestic mild cognitive impairment. Dement Geriatr Cogn Disord. 2011 Apr;31(3):225-32.

28 Ihle A, Gouveia ÉR, Gouveia BR, Freitas DL, Jurema J, Ornelas RT, et al. The relation of education and cognitive activity to minimental state in old age: the role of functional fitness status. Eur J Ageing. 2018 Jun;15(2): 123-31.

29 Ihle A, Gouveia ÉR, Gouveia BR, Freitas DL, Jurema J, Odim AP, et al. The relation of education, occupation, and cognitive activity to cognitive status in old age: the role of physical frailty. Int Psychogeriatr. 2017 Sep;29(9): 1469-74.

30 Ihle A, Zuber S, Gouveia ÉR, Gouveia BR, Mella N, Desrichard O, et al. Cognitive reserve mediates the relation between openness to experience and smaller decline in executive functioning. Dement Geriatr Cogn Disord. 2019 Sep;48(1-2):39-44
31 Oris M, Guichard E, Nicolet M, Gabriel R, Tholomier A, Monnot C, et al. Representation of vulnerability and the elderly. In: Oris M, Roberts C, Joye D, Ernst-Stähli M, editors. Surveying human vulnerabilities across the life course. Heidelberg: Springer; 2016. p. 27 64.

32 Ludwig C, Cavalli S, Oris M. "Vivre/Leben/ Vivere": an interdisciplinary survey addressing progress and inequalities of aging over the past 30 years in Switzerland. Arch Gerontol Geriatr. 2014 Sep-Oct;59(2):240-8.

33 Ihle A, Grotz C, Adam S, Oris M, Fagot D, Gabriel R, et al. The association of timing of retirement with cognitive performance in old age: the role of leisure activities after retirement. Int Psychogeriatr. 2016 Oct;28(10): 1659-69.

34 Ihle A, Oris M, Fagot D, Chicherio C, van der Linden BWA, Sauter J, et al. Associations of educational attainment and cognitive level of job with old age verbal ability and processing speed: the mediating role of chronic diseases. Appl Neuropsychol Adult. 2018 Jul-Aug; 25(4):356-62.

35 Ihle A, Oris M, Fagot D, Kliegel M. The relation of the number of languages spoken to performance in different cognitive abilities in old age. J Clin Exp Neuropsychol. 2016 Dec; 38(10):1103-14.

36 Ihle A, Mons U, Perna L, Oris M, Fagot D, Gabriel R, et al. The relation of obesity to performance in verbal abilities, processing speed, and cognitive flexibility in old age: the role of cognitive reserve. Dement Geriatr Cogn Disord. 2016 Sep;42(1-2):117-26.

37 Ihle A, Oris M, Sauter J, Rimmele U, Kliegel M. Cognitive reserve and social capital accrued in early and midlife moderate the relation of psychological stress to cognitive performance in old age. Dement Geriatr Cogn Disord. 2018 Jun;45(3-4):190-7.

38 Ihle A, Gouveia ÉR, Gouveia BR, Zuber S, Kliegel M. Cognitive reserve attenuates the relation between gastrointestinal diseases and subsequent decline in executive functioning. Dement Geriatr Cogn Disord. 2019;48(3-4): 215-8.

39 Ihle A, Gouveia ÉR, Gouveia BR, Cheval B, Sieber S, Cullati S, et al. Cognitive reserve attenuates 6-year decline in executive functioning after stroke. Dement Geriatr Cogn Disord. 2019;48(5-6):349-53.

40 Reitan RM. Validity of the trail making test as an indicator of organic brain damage. Percept Mot Skills. 1958 Dec;8(7):271-6.

41 Lalive d'Epinay C, Bickel JF, Hagmann HM, Maystre C, Michel JP. Comment définir la grande vieillesse? Du recours à l'âge chronologique ou à l'âge socio-fonctionnel. [How to define oldest old? Using chronological age or socio-functional age.] L'année gérontologique [Gerontological Yearbook]. 1999. Vol. 13; p. 64-83. 
42 Lalive d'Epinay C, Pin S, Spini D. Présentation de SWILSO-O, une étude longitudinale suisse sur le grand âge: l'exemple de la dynamique de la santé fonctionnelle. 2001. Vol. 15; p. 78-96.

43 Nucci M, Mapelli D, Mondini S. Cognitive reserve index questionnaire (CRIq): a new instrument for measuring cognitive reserve. Aging Clin Exp Res. 2012 Jun;24(3):218-26.

44 Vallet F, Mella N, Ihle A, Beaudoin M, Fagot $\mathrm{D}$, Ballhausen N, et al. Motivation as a mediator of the relation between cognitive reserve and cognitive performance. J Gerontol B Psychol Sci Soc Sci. 2020 Jun;75(6):1199-205.
45 Ihle A, Ghisletta P, Ballhausen N, Fagot D, Vallet F, Baeriswyl M, et al. The role of cognitive reserve accumulated in midlife for the relation between chronic diseases and cognitive decline in old age: a longitudinal follow-up across six years. Neuropsychologia. 2018 Dec; 121:37-46.

46 Ihle A, Bavelier D, Maurer J, Oris M, Kliegel $\mathrm{M}$. Internet use in old age predicts smaller cognitive decline only in men. Sci Rep. 2020 Jun;10(1):8969.

47 Chen JH, Chan DC, Kiely DK, Morris JN, Mitchell SL. Terminal trajectories of functional decline in the long-term care setting. J Gerontol A Biol Sci Med Sci. 2007 May;62(5): 531-6.
48 Lunney JR, Lynn J, Foley DJ, Lipson S, Guralnik JM. Patterns of functional decline at the end of life. JAMA. 2003 May;289(18):2387-92.

49 Aartsen MJ, Cheval B, Sieber S, Van der Linden BW, Gabriel R, Courvoisier DS, et al. Advantaged socioeconomic conditions in childhood are associated with higher cognitive functioning but stronger cognitive decline in older age. Proc Natl Acad Sci U S A. 2019 Mar; 116(12):5478-86.

50 Zuber S, Ihle A, Blum A, Desrichard O, Kliegel $\mathrm{M}$. The effect of stereotype threat on age differences in prospective memory performance: differential effects on focal versus nonfocal tasks. J Gerontol B Psychol Sci Soc Sci. 2019 Apr;74(4):625-32. 